\title{
Problemas éticos encontrados en la asistencia sanitaria y toma de decisiones que involucran a personas que viven con demencia y otros impedimentos cognitivos progresivos \\ Ethical issues encountered in healthcare decision making involving people living with dementia and other progressive cognitive impairments
}

\author{
Julian C. Hughes*
}

https://doi.org/10.36105/mye.2020v31n4.05

\section{Resumen}

Este artículo discute los problemas relacionados con la toma de decisiones en la demencia (y otras deficiencias cognitivas progresivas). Presenta cinco de estos temas; a saber, los relacionados con el lugar de residencia, la atención forzada, la medicación encubierta (en relación con la cual también mencionaré la verdad), la sexualidad, la comida y la bebida, que incluye cierta consideración de suspender y retirar el tratamiento en general. Después reflexionará sobre cómo se trata la toma de decisiones en la práctica clínica; posteriormente discutirá algunos temas con-ceptuales: capacidad (o competencia), mejores intereses y toma de decisio-

\footnotetext{
* Profesor honorario, Universidad de Bristol, Reino Unido. Profesor visitante, Universidad de Newcastle, Reino Unido. Correo electrónico: julian.hughes@ bristol.ac.uk https://orcid.org/0000-0002-9863-0478

Recepción: 20 de junio de 2020. Aceptación: 15 de julio de 2020.
} 
nes con apoyo. Terminará con algunos comentarios sobre la importancia de la individualidad en relación con los problemas éticos que rodean la toma de decisiones en la demencia.

Palabras clave: mejores intereses, capacidad, individualidad, verdad, toma de decisiones con apoyo, ética médica.

\section{Introducción}

Quizás sea mejor decir lo que este documento no es: no es una revisión de todos los problemas éticos que ocurren en relación con la toma de decisiones respecto de personas que viven con demencia y otras deficiencias cognitivas progresivas. También debo resaltar que, a partir de ahora, sólo hablaré de personas que viven con demencia, a pesar de que hay una variedad de otras condiciones progresivas que pueden conducir a trastornos cognitivos. Esto se debe principalmente a la brevedad, pero también porque estoy más familiarizado con los problemas relacionados con la demencia, aunque son esencialmente los mismos. Sin embargo, ciertamente no lo es, porque la toma de decisiones en otras condiciones debe ser ignorada.

He escogido cinco cuestiones. No se seleccionan completamente al azar. Son de actualidad o son temas en los que estoy más familiarizado con la literatura. Luego paso a discutir, aunque sea brevemente, temas conceptuales de fondo que son relevantes para todo tipo de debates y dilemas de toma de decisiones. Finalmente, concluiré con algunas reflexiones generales sobre el tema de la personalidad en la demencia. Para comenzar, deseo resaltar la variedad de cuestiones éticas relevantes para vivir con demencia.

\section{Problemas éticos en la demencia}

Hace algunos años participé en una investigación que buscaba cuestiones éticas para los cuidadores de personas que viven con 
Problemas éticos encontrados en la asistencia sanitaria y toma de decisiones...

demencia. Deseo señalar tres puntos de esa investigación. Primero, el investigador no preguntó específicamente sobre cuestiones «éticas», sino más bien sobre problemas y dificultades. Sin embargo, más o menos todo lo mencionado resultó tener un componente ético. En segundo lugar, la variedad de temas mencionados fue amplia (ver Tabla 1), yendo mucho más allá del tipo de problemas que tradicionalmente se debatirían en un libro de texto de ética. Tercero, casi todos los problemas tenían que ver con la toma de decisiones. Después de todo, esto no debería ser una gran sorpresa, dado que una característica clave de la demencia es que la persona pierde gradualmente la capacidad de decidir por sí misma.

Más recientemente, Strech, por ejemplo (3), realizó una revisión cualitativa sistemática de la literatura inglesa y alemana para identificar el espectro completo de problemas éticos en la demencia. Iden-

Tabla 1. Problemas éticos para cuidadores de personas que viven con demencia [derivados de] (1) y (2).

\begin{tabular}{|l|l|l|}
\hline \multicolumn{1}{|c|}{ Evaluación } & \multicolumn{1}{|c|}{$\begin{array}{c}\text { Comportamiento } \\
\text { desafiante }\end{array}$} & \multicolumn{1}{c|}{ Comunicación } \\
\hline Recursos de la comunidad & Confidencialidad & Consentimiento \\
\hline Restricción & Conducción & Doble lealtad \\
\hline Fin de la vida & Eutanasia & Finanzas \\
\hline Prueba genética & Renunciar a la atención & Culpa \\
\hline Infantilización & Falta de apoyo & Decepcionándolos \\
\hline Cuidado de largo plazo & Medicación & Malentendido \\
\hline Necesidad de informacion & Personal care & Profesionalismo \\
\hline Vergüenza pública & Relaciones con los doctores & Cuidado de relevo \\
\hline Cuidados personales & La privación del sueño & Asumir tareas \\
\hline Tomar riesgos & Hablando de la persona & Llamadas telefónicas \\
\hline Informando el diagnóstico & Tratar a la persona & $\begin{array}{l}\text { Decisiones de } \\
\text { tratamiento }\end{array}$ \\
\hline Diciendo la verdad & Vulnerabilidad & Ambulante \\
\hline
\end{tabular}

Fuente: Elaboración propia.

Medicina y Ética - Octubre-Diciembre 2020 - Vol. 31 - Núm. 4 
tificaron 56 problemas éticos específicos de la demencia, que se agruparon en siete categorías principales:

- Diagnóstico e indicación médica.

- Evaluar la capacidad del paciente para la toma de decisiones.

- Información y divulgación.

- Toma de decisiones y consentimiento.

- Aspectos sociales y contextuales.

- Proceso de atención y evaluación del proceso.

- Situaciones especiales, que incluyen: capacidad para conducir, relaciones sexuales, indicación para pruebas genéticas, uso de GPS (Sistema de Posicionamiento Global) y otras técnicas de monitoreo, prescripción de antibióticos o de medicamentos antipsicóticos, medicación encubierta, restricciones, alimentación por sonda y finalización de la vida o cuidados paliativos.

Resulta evidente que dos de las principales categorías tienen que ver abiertamente con la toma de decisiones, y casi todas las situaciones especiales implican también la toma de decisiones. Por tanto, el tema del cuidado de la demencia es amplio y muy real. Analizaré sólo cinco de sus aspectos, pero de manera que arroje algo de luz también sobre otros temas. Destacaré en las discusiones que siguen la importancia central de la noción de personalidad como tema subyacente, y la volveré a señalar en mi conclusión.

\section{Cinco cuestiones}

\section{a) Lugar de residencia}

Para ilustrar cada uno de estos temas, comenzaré con unos ejemplos ficticios. Son escasos en términos de detalle; sólo pretenden orientarnos en el problema específico. 
Problemas éticos encontrados en la asistencia sanitaria y toma de decisiones...

El señor Bentley ingresó en el hospital con una confusión causada por una infección de orina. La infección fue tratada, pero él permaneció confundido. Los vecinos y la familia informan que ha estado «deambulando» por la calle en las tardes durante un tiempo. ¿Es seguro, por lo tanto, que él se vaya a casa, donde vivió con su esposa durante los 50 años de su matrimonio?

Esto plantea el problema de la capacidad de residencia: si las personas tienen la capacidad de tomar decisiones sobre dónde viven. Es un problema muy real de todos los días en los principales hospitales. Las personas mayores como el señor Bentley podrían simplemente tener una confusión aguda, un delirio, que se resolvería con más tiempo; pero él podría tener demencia. Incluso si tiene demencia, aún podría ser posible para él vivir en casa con un tipo de apoyo adecuado. Aun así, ahora corre el riesgo de que se considere que carece de la capacidad o competencia para tomar esta decisión, en cuyo caso podría ser llevado a vivir a un hogar de ancianos en contra de su voluntad, con pocas posibilidades de regresar a su propio hogar y con todos sus recuerdos.

En el Reino Unido ha habido una serie de casos judiciales en torno a este tema. La Cámara de los Lores examinó, en 2014, cómo funcionaba la ley que rige la capacidad de toma de decisiones en Inglaterra y Gales -la Ley de Capacidad Mental de 2005- (4). Aunque la ley tenía buenas intenciones, sintieron que no se estaba implementando correctamente. Es probable que una de sus conclusiones fuese relevante en otros países y jurisdicciones: «las culturas prevalecientes de paternalismo (en salud) y aversión al riesgo (en atención social) han impedido que la Ley sea ampliamente conocida o incorporada». En otras palabras, debido a que los médicos tienden a sentir que deben proteger a sus pacientes, como el señor Bentley, y a que los trabajadores sociales tienden a evitar riesgos y acciones legales por parte de sus familiares, la opción más fácil será colocarlo en un centro de asistencia (5). Sin embargo, para el señor Bentley esto podría sentirse como un gran golpe a su autoestima: como si él y sus sentimientos ya no fueran importan- 
tes. Está indefenso ante la presión profesional y social para mantenerlo a salvo.

\section{b) Cuidados forzados}

La señora Jones, con notable demencia, es doblemente incontinente, pero se niega a dejar que alguien la cambie cuando se ensucia. Es preocupante para el personal saber que está muy sucia, y los residentes y sus familias están comenzando a quejarse. Esto lleva a que la señora Jones sea rechazada $y$, de hecho, ha habido una creciente agresión verbal hacia ella, con personas que la insultan. El personal tiene diferencias de opinión sobre lo que deben hacer.

Esta puede ser una decisión muy delicada para los cuidadores y las familias. La señora Jones desea mantener el control de su vida, especialmente su vida personal, pero parece que ahora carece de una perspectiva de la naturaleza de sus problemas y los efectos que están teniendo en los demás. Para el personal, no forzar la solución (suponiendo que hayan usado toda su habilidad para hacer que se lave o que se le ayude de una manera amable) supone arriesgar su seguridad (es decir, el riesgo de infecciones o el riesgo de agresión de otros residentes) y socavar su dignidad. Sin embargo, usar la fuerza puede hacer sentir al personal (y a otros) como si la estuvieran agrediendo. Desnudarla contra su voluntad parece un abuso y mina su posición como persona. El personal, a menudo, requerirá una buena cantidad de apoyo para alentarlos a intervenir de manera segura y legal para proteger a la señora Jones (6).

\section{c) Medicación encubierta y decir la verdad}

Joseph Leblanc, ex director gerente de una empresa de transporte canadiense, no cree que haya nada malo en él. Además de la demencia, tiene insuficiencia cardiaca y, si no toma su medicamento, se queda sin aliento progresivamente, muy angustiado y, a veces, agresivo. El personal del asilo descubrió que si le ponen el medicamento en el budín, se lo come sin quejarse y se mantiene estable. Cuando está estable, está 
Problemas éticos encontrados en la asistencia sanitaria y toma de decisiones...

contento y parece disfrutar la vida. Un nuevo miembro del personal siente que el uso de medicamentos encubiertos es engañoso e incorrecto.

Aquí hay dos cuestiones relacionadas: la de ocultar la medicación y las circunstancias (si las hay) cuando esto está justificado, y el tema más amplio de decir la verdad. Muchos países ahora tendrán orientación, especialmente para las enfermeras, sobre las circunstancias bajo las cuales los medicamentos pueden ocultarse en los alimentos y bebidas, generalmente como último recurso y sólo si están debidamente documentados. El problema, sin embargo, es ciertamente ético (7).

Mentir a las personas con demencia también es un problema importante. Toma muchas formas y tipos, desde no decir la verdad sobre el diagnóstico, hasta el engaño sobre la atención que se proporcionará a la persona (por ejemplo, ir a un asilo); desde decirle a la persona que un pariente en particular todavía está vivo, cuando murió hace algún tiempo, hasta proporcionar indicios ambientales falsos (por ejemplo, una parada de autobús falsa en la que la persona espera con la ilusoria promesa de que irá a algún lado). A veces, la verdad absoluta puede parecer dolorosa, pero una mentira descarada parece insultante para la persona.

En un informe sobre este tema, la Fundación de Salud Mental (8) ideó un continuum, como se muestra en la Figura 1. La sugerencia es que siempre se debe empezar lo más cerca posible de decir la verdad. Si hay que desviarse de esto, debe hacerlo sólo por buenas razones y se debe continuar con pasos ligeros, a menos que haya

Figura 1. Un continuo de toda la verdad hasta las mentiras flagrantes (8).

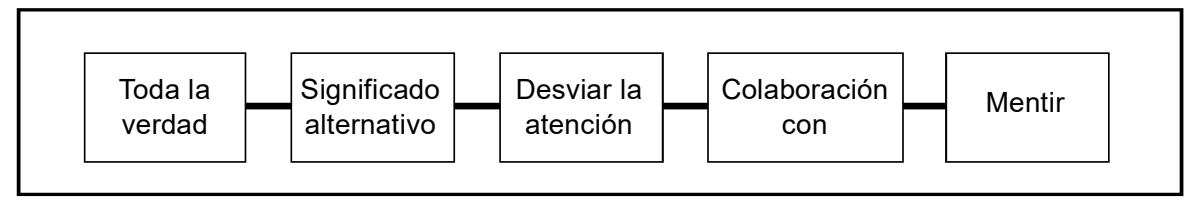

Fuente: Elaboración propia.

Medicina y Ética - Octubre-Diciembre 2020 - Vol. 31 - Núm. 4 
buenas razones para no hacerlo. Por ejemplo, si ya se sabe que habrá una reacción catastrófica, por ejemplo, a «buscar un significado alternativo» (es decir, tratar de comprender las emociones que se están expresando), entonces sería mejor usar una «distracción» inmediatamente. Aun así, la mejor práctica es que el personal debe mantener planes de atención para las decisiones que impliquen cualquier forma de engaño.

\section{d) Sexualidad}

La señora Gauthier era nueva en el asilo para personas con demencia avanzada, pero a los pocos días había entablado una amistad con el señor Wong. Ambos estaban casados y sus cónyuges los visitaban a diario. Sus parejas quedaron desconcertados al descubrir que la relación parecía profundizarse. Católicos devotos, ambos siempre habían sido fieles a sus respectivos cónyuges. Después de tres meses, los miembros del personal tuvieron que informar a las familias que el señor Wong había sido encontrado desnudo con la señora Gauthier en su cama. La señora Wong está molesta, pero acepta que su esposo necesita compañía. El señor Gauthier está furioso y afirma que el señor Wong es un depredador y un viejo verde que debe ser expulsado del asilo.

La sexualidad en las personas mayores es propensa a provocar actitudes contra la tercera edad, las cuales se ven agravadas por el estigma cuando se trata de personas que viven con demencia. En casos como los de la señora Gauthier y el señor Wong, la reacción inicial a menudo puede ser legal, y también puede ser sexista. A menudo el hombre es visto como el perpetrador $y$, dado que surge una pregunta sobre la capacidad de la mujer para dar su plenamente informado consentimiento, a menudo se alega que tales casos equivalen a violación. Por lo tanto, la policía está informada y los asuntos se vuelven muy molestos para todos.

Después de todo, puede ser que haya ocurrido una violación. Pero también puede ser que el señor Wong realmente crea que la señora Gauthier es su esposa, y tal vez ella inició la actividad sexual. Ambos podrían creer que son una pareja joven, soltera, y estar 
Problemas éticos encontrados en la asistencia sanitaria y toma de decisiones...

bastante sorprendidos por el alboroto que se está haciendo. Un problema en sí mismo es cómo se evalúa la capacidad de consentimiento en tales situaciones, especialmente si los residentes en el hogar tienen dificultades de comunicación. Los problemas relacionados con la sexualidad también incluyen las relaciones entre homosexuales y lesbianas, y las actitudes del personal y otros residentes hacia la homosexualidad o las cuestiones de transgénero, etcétera.

$\mathrm{Si}$, por un lado, tales dilemas se ven a través de una lente legal, por otro también tienen que ver con la intimidad y la necesidad humana de consuelo y relaciones cercanas y tranquilizadoras (9). Sin embargo, la realidad es que nuestras respuestas a la sexualidad e intimidad humanas, que incluirán actitudes hacia la masturbación, la pornografía, los fetiches, etc., son variadas. Sabemos que la sexualidad y la necesidad de intimidad son intrínsecas a nuestra naturaleza como seres humanos, pero en los grupos vulnerables, inevitablemente habrá cierta precaución al permitir ciertos tipos de actividad. El dilema ético, como suele ser el caso, es decidir en casos particulares dónde se deben trazar las líneas para permitir la plena expresión de la sexualidad y la intimidad, pero sin permitir que ocurra el abuso.

e) Alimentos y bebidas (retener y retirar)

Miss Cote se encuentra en las etapas severas de la demencia. Su deglución se está volviendo cada vez más comprometida. Ella se ahoga y ya ha tenido varias infecciones en el pecho. Vive en casa y tiene cuidadores que vienen a alimentarla, pero esto se lleva más y más tiempo. Su médico de cabecera (MC) le sugiere a la familia que se le debe colocar un tubo de gastrostomía endoscópica percutánea (GEP) para colocar la comida y la bebida directamente en su estómago. Esto requerirá un ingreso hospitalario. Su familia prefiere, cualesquiera que sean los riesgos, que continúe siendo alimentada a mano. Sus cuidadores, sin embargo, están alarmados por su asfixia.

Casos como los de Miss Cote no son inusuales. Nuevamente, el problema ético es real. Es la cuestión de retener o retirar investiga- 
ciones o tratamientos. Cuestiones similares surgen en diversas circunstancias en la demencia, no sólo en relación con la deglución, sino también con respecto al uso de antibióticos o en torno a la reanimación cardiopulmonar (10).

Sin embargo, existe una doctrina bien establecida, la de los medios ordinarios y extraordinarios, que puede ser útil para enfrentar estos dilemas. La doctrina puede expresarse de la siguiente manera: no estamos moralmente obligados a llevar a cabo una investigación o un tratamiento que puede considerarse como extraordinario. $\mathrm{Y}$ es extraordinario si es poco probable que funcione y si es oneroso para el paciente.

En el caso de Miss Cote podemos decir que es poco probable que el tubo GEP logre sus objetivos (11), y es oneroso porque implica un anestésico y una variedad de riesgos (por ejemplo, infección y sangrado). Por lo tanto, puede considerarse como un tratamiento extraordinario y no debería existir una obligación moral de buscar el uso de alimentación PEG en este caso. Se puede utilizar un razonamiento similar para la reanimación cardiopulmonar. El uso de antibióticos en la demencia severa es más complejo porque es más discutible si los antibióticos son eficaces o no. Porque, incluso, si no efectúan una cura, pueden usarse de forma paliativa (12). Sin embargo, si con su uso fuera necesario ingresar al hospital para colocar una vía intravenosa, también podría considerarse demasiado oneroso para un determinado paciente en particular.

\section{Temas conceptuales}

Ahora consideraré tres temas conceptuales planteados por los casos discutidos anteriormente.

El primer tema tiene que ver con la posición de las evaluaciones de capacidad como pruebas objetivas. Hablamos habitualmente de evaluaciones de la capacidad de una persona (llamada competencia en algunas jurisdicciones). Un error que los médicos tienden a co- 
Problemas éticos encontrados en la asistencia sanitaria y toma de decisiones...

meter es considerar que la capacidad depende del resultado de cualquier decisión que se tome. Por lo tanto, si una persona toma una decisión que parece segura, se dice que tiene capacidad (si se satisfacen otros criterios). Si, como el señor Bentley en el caso descrito anteriormente, quiero irme a casa a pesar de que existen riesgos asociados con esta opción, es muy posible que carezca de la capacidad necesaria para tomar esta decisión. Esta tendencia, a favor de un enfoque de resultados en lugar de uno funcional (es decir, uno que analiza el funcionamiento cognitivo involucrado en la toma de decisiones independientemente de los resultados elegidos), refleja la tendencia a hacer lo mejor para los pacientes, donde esto tiende a significar mantenerlos a salvo (13). En sí, esta es una decisión de evaluación: implica un juicio de valor, en este caso, uno que es erróneo. Sin embargo, incluso si se evita cometer el error de una evaluación basada en el resultado, en lugar de las habilidades funcionales, es probable que los juicios sobre la capacidad siempre contengan un elemento normativo (14). Para que una persona tenga la capacidad, ¿cuánta información debería tener en cuenta, y por cuánto tiempo podría constituir una ponderación suficiente de esa información? Es una pretensión que estos criterios son totalmente objetivos.

El segundo tema es el de los mejores intereses. El punto aquí es que, si vamos a emitir juicios sobre lo que es mejor para una persona, debemos tener una visión lo más amplia posible. En Inglaterra y Gales, la Ley de Capacidad Mental de 2005 (sección 4) establece una lista de verificación para los mejores intereses, que parece muy sensata:

- General:

- Evitar la discriminación;

- Considerar todas las circunstancias relevantes.

- Pasos particulares:

- Evaluar si la persona podría recuperar la capacidad en un futuro próximo;

- Fomentar la participación; 
- Evitar estar motivado de alguna manera por el deseo de provocar la muerte de la persona.

- Considerar lo más ampliamente posible:

- Los deseos, sentimientos, creencias y valores de la persona;

- Tener en cuenta las opiniones de quienes cuidan a la persona.

Esta amplia visión refleja directamente la visión extensa necesaria para caracterizar la personalidad misma.

El último tema es el de la toma de decisiones con apoyo, que surge de la Convención de las Naciones Unidas sobre los Derechos de las Personas con Discapacidad (CNUDPD) (15). La noción de toma de decisiones con apoyo tiene como objetivo señalar que sigue siendo la decisión de la persona, incluso si está muy discapacitada (inclusive con demencia). Una decisión sustituida es esencialmente una decisión tomada por otra persona para la persona; e incluso una decisión compartida suena como si alguien diferente a la persona juega un papel demasiado grande. El problema ha sido bien planteado por Scholten y Gather (16):

A pesar de su promesa, la TDA (Toma de Decisiones con Apoyo) tiene sus límites. Sin lugar a dudas, hay situaciones en las que, a pesar del apoyo de decisión ofrecido, un paciente sigue sin poder tomar una decisión de tratamiento informada. El paciente comatoso proporciona un ejemplo pertinente, pero existen consideraciones similares para una variedad de personas con otras afecciones, como la demencia en etapa tardía o la psicosis. Por tanto, la TDA no hace superflua la evaluación de competencias y sustituye la toma de decisiones. Dicho esto, una adaptación razonable requiere que los profesionales de la salud agoten los recursos disponibles de la TDA antes de recurrir a sustituir la toma de decisiones.

$\mathrm{El}$ argumento es que la persona debe ser puesta en primer lugar, lo cual es, después de todo, el llamado a la atención centrada en la persona con demencia (17). 
Problemas éticos encontrados en la asistencia sanitaria y toma de decisiones...

\section{Conclusión}

Cada uno de los temas brevemente mencionados anteriormente nos involucra a pensar en las personas y en lo que somos como personas. En pocas palabras, plantean problemas sobre la personalidad. La atención a la personalidad implica valores y hacer juicios evaluativos (18). Se requieren juicios amplios para decidir qué es lo mejor para la persona, porque lo que es ser una persona debe ser ampliamente concebido. La toma de decisiones con apoyo es importante precisamente porque enfatiza la posición de las personas, incluso cuando viven con discapacidades profundas. A su vez, los cinco temas que reflexionamos anteriormente también requieren que pensemos profundamente acerca de la persona, la cual debería ocupar un lugar central cuando deben tomarse estas decisiones difíciles.

Según mi manera de pensar, la forma adecuada de caracterizar la personalidad de las personas con demencia es considerarlas como agentes encarnados situados $(19,20)$. Están situados en una variedad de campos, involucrando sus propias narrativas y las de otros, así como también en los campos culturales, sociales, legales, normativos, etc. De hecho, las formas en que se sitúan las personas no pueden circunscribirse. Mientras tanto, las personas están encarnadas: no se puede ignorar su naturaleza corporal y la forma en que se expresan corporalmente. Finalmente, son agentes y continúan teniendo inclinaciones de agente, incluso cuando viven con demencias severas. La toma de decisiones en la demencia es una cuestión ética, precisamente porque la tentación es tomar el camino fácil y socavar la posición de la persona con demencia. Esta es una tentación a la que debemos resistir, si queremos comportarnos como florecientes seres humanos morales.

Medicina y Ética - Octubre-Diciembre 2020 - Vol. 31 - Núm. 4 


\section{Referencias bibliográficas}

1. Hughes JC, Hope T, Savulescu J, Ziebland S. Cuidadores, ética y demencia: una encuesta y revisión de la literatura. Int J Geriatr Psychiatry. 2002; 17 (1): 35-40.

2. Hughes JC, Hope T, Reader S, Rice D. Demencia y ética: las opiniones de los cuidadores informales. J Roy Soc Med. 2002; 95(50): 242-246.

3. Strech D, Mertz M, Knüppel H, Neitzke G, Schmidhuber M. El espectro completo de cuestiones éticas en el cuidado de la demencia: revisión cualitativa sistemática. Br J Psychiatry. 2013; 202(6): 400-406. https://doi.org/10.1192/bjp.bp.112. 116335

4. Cámara de los Lores. Ley de capacidad mental de 2005: escrutinio post-legislativo. Londres: The Stationery Office Limited; 2014.

5. Poole M, Bond J, Emmett C, Greener H, Louw SJ, Robinson L, Hughes JC. ¿Ir a casa? Un estudio etnográfico de evaluación de la capacidad y los mejores intereses en personas con demencia que reciben el alta hospitalaria. Bmc Geriatr. 2014; 14: 56. [consultado el 11 de junio de 2020]. https://doi.org/10.1186/1471-23 18-14-56

6. Howarth A, Sells D, Mackenzie L, Hope A. ¿Estamos obligando a las personas con demencia a recibir atención? Int J Geriatr Psychiatry. 2014; 29(7): 767-770.

7. Treloar A, Philpot M, Beats B. Ocultar medicamentos en la comida de los pacientes. Lancet. 2001; 357(9265): 62-64.

8. Williamson T, Kirtley A. ¿Qué es la verdad? Una investigación sobre la verdad, la mentira, las diferentes realidades y creencias en el cuidado de la demencia (revisión de evidencia e informe). Londres: Fundación de Salud Mental; 2016. https:// doi.org/10.2307/j.ctvb6v5hs.10

9. Hughes JC, Beatty A, Shippen J. Sexualidad en la demencia. En: Foster C, Herring J, Doron I, editores. La ley y la ética de la demencia. Oxford: Hart Publishers; 2014: 227-238.

10. Hughes JC, Baldwin C. Cuestiones éticas en el cuidado de la demencia: tomar decisiones difíciles. Londres y Filadelfia: Jessica Kingsley; 2006.

11. Sampson EL, Candy B, Jones L. Alimentación por sonda enteral para personas mayores con demencia avanzada. Base de datos Cochrane Syst Rev. 2009 [consultado el 11 de junio de 2020]. Tema 2, Art. No. CD007209. http://dx.doi.org/ 10.1002/14651858.CD007209.pub2

12. Van Der Steen JT, Roeline H, Pasman W, Ribbe MW, Van der Wal G, Onwuteaka-Philipsen BD. Molestias en pacientes con demencia que mueren de neumonía y su alivio con antibióticos. Scand J Infect Dis. 2009; 41(2): 143-151. https:// doi.org/10.1080/00365540802616726

13. Greener H, Poole M, Emmett C, Bond J, Louw SJ, Hughes JC. Juicios de valor y tensiones conceptuales: toma de decisiones en relación con el alta hospitalaria para personas con demencia. Clin Ethics. 2012; 7(4): 166-174.

14. Charland LC. Competencia mental y valor: el problema de la normatividad en la evaluación de la capacidad de toma de decisiones. Psychiat Psychol Law. 2001; 8(2): 135-145. https://doi.org/10.19083/tesis/624270 
Problemas éticos encontrados en la asistencia sanitaria y toma de decisiones...

15. Convención de las Naciones Unidas sobre los Derechos de las Personas con Discapacidad. Ginebra: Naciones Unidas; 2006. [Consultado el 11 de junio de 2020]. Disponible en: https://www.un.org/development/desa/disabilities/convention-on-the-rights-of-persons-with-disabilities.html https://doi.org/10.22201/iij.2448 7872e.2008.8.252

16. Scholten M, Gather J. Consecuencias adversas del artículo 12 de la Convención de las Naciones Unidas sobre los derechos de las personas con discapacidad para las personas con discapacidad mental y un camino alternativo a seguir. J Med Ethics. 2018; 44(4): 226-233. https://doi.org/10.2139/ssrn.2037464

17. Kitwood T. Brooker D, editor. La demencia se reconsidera, se revisa: la persona sigue siendo lo primero. $2^{\mathrm{a}}$ ed. Londres: Open University Press; 2019.

18. Hughes JC, Williamson T. El manifiesto de la demencia: llevar a cabo la práctica basada en valores. Cambridge: Cambridge University Press; 2019.

19. Hughes JC. Opiniones de la persona con demencia. J Med Ethics. 2001; 27 (2): 86-91.

20. Hughes JC. Pensando en la demencia. Oxford: Oxford University Press; 2011. 
